Alexander Merk, OMS III, Emily Nelson, OMS III, Alisha Provost, OMS IV, Matthew Rasch, OMS IV, Lisa Forster, MS, Kelly Nottingham, MPH, Janet Simon, PhD and Todd Fredricks*, DO

\title{
A survey on health professionals' understanding of federal protections regarding service dogs in clinical settings
}

https://doi.org/10.1515/jom-2020-0280

Received November 12, 2019; accepted September 4, 2020;

published online February 10, 2021

\begin{abstract}
Context: Research has been scarce on health professionals' knowledge about guidelines regulating service dogs in a clinical setting. Gaining insight into health professionals' understanding of Americans with Disabilities Act (ADA) regulations concerning service dogs is critical for navigating compliance and reducing risk. Misinformation about service dogs could influence decisions affecting policy and care, leading to poor treatment and suboptimal health outcomes for patients with service animals.

Objectives: To assess health professionals' knowledge about ADA regulations and beliefs about workplace protocols and training related to service dogs.

Methods: The study used snowball sampling to distribute surveys to health professionals from around the United States. Initial outreach occurred using mailing lists, investigators' personal networks, and social media. The survey contained 24 items. True and false questions were used to test ADA knowledge and then coded as correct or incorrect. Most closed-end questions were measured on a 5-point Likert scale using frequencies and descriptive statistics. A one-way analysis of variance (ANOVA) was conducted to test whether variables, such as encounters to service dogs, affected knowledge of ADA requirements.
\end{abstract}

\footnotetext{
*Corresponding author: Todd Fredricks, DO, Heritage College of Osteopathic Medicine, Athens, OH, USA, E-mail: fredrick@ohio.edu. https://orcid.org/0000-0001-8466-2834

Alexander Merk, OMS III, Emily Nelson, OMS III, Alisha Provost, OMS IV, Matthew Rasch, OMS IV and Lisa Forster, MS, Heritage College of Osteopathic Medicine, Athens, OH, USA

Kelly Nottingham, MPH, Ohio University Graduate College, Athens, $\mathrm{OH}$, USA

Janet Simon, PhD, College of Health Sciences and Professions, Athens, OH, USA
}

Results: The survey was completed by 441 health professionals from around the country. Most (234; 53.1\%) worked in a hospital and came from a range of professional backgrounds (nurses, 155 [35.2\%]; physicians, 71 [16.1\%]). While nearly three-quarters (318 [73.1\%]) of participants said their workplace had a policy on service animals, 113 (34.9\%) of those said they were unfamiliar with the policy and $236(54.5 \%)$ said they had not received adequate training on the topic. Most participants did not know basic ADA policy requirements related to service dogs. Only those who were extremely familiar with policy $(F=4.613 ; \mathrm{p}=0.001)$ and those who strongly agreed that they knew the differences between service dogs and other classes of animals ( $F=5.906$; $\mathrm{p}=0.000$ ) scored higher on the knowledge test than those who disagreed.

Conclusions: Our results suggest that increased familiarity and training leads to higher knowledge about service dogs and ADA policy. Health professionals need additional education on ADA service dog regulations and hospital policy in order to minimize risk and ensure patients with service dogs receive optimal care.

Keywords: ADA; hospital policy; regulations; service dogs.

Most existing literature on service animals has examined how they affect owners' health outcomes, but there has been little research on health professionals' understanding of federal regulations and protections for patients who own service dogs [1-4]. Gaining insight on this issue is critical because misinformation about service dogs could affect policy decisions, ultimately leading to poor treatment and suboptimal health outcomes for patients with service animals. In the past decade, the use of service animals to support individuals with health issues has grown considerably in the United States [5]. A 2017 study [4] showed wide public misunderstanding around the definitions, rights, and rules of service dogs [4]. One key issue involves 
how a service animal is legally defined. According to the Americans with Disabilities Act (ADA), service animals are "dogs that are individually trained to do work or perform tasks for people with disabilities" [6]. This includes assisting people who are blind, deaf, have mobility difficulties, mental illness, or post-traumatic stress. The definition excludes emotional support animals (which offer comfort but have not been trained to perform a specific task for someone who is disabled) and therapy dogs (which provide comfort and affection to individuals, disabled or not, in schools, health facilities and similar locations). The ADA lists examples of tasks performed by service dogs, such as reminding a person to take medicine or pulling a wheelchair [6]. Dogs typically undergo intensive training, which can cost $\$ 30,000-\$ 50,000$, to learn these skills [7]. To further clarify the role, the ADA states that a service dog is not a pet, but a working animal [6]. The distinction can be difficult to make. According to a 2017 survey by the American Veterinary Medical Association, there are about 77 million pet dogs in the U.S. and $85 \%$ of pet owners consider dogs to be family members [8]. A 2016 survey found that $19 \%$ of dog owners have sneaked them into places where dogs were not allowed [9].

Service dogs, in particular fake service dogs, have received considerable media attention highlighting individuals who have tried to circumvent no pet policies in apartments, restaurants, and on airlines by claiming they have a service dog [10-15]. Numerous organizations sell paraphernalia like vests and certifications, which make that type of fraud harder to detect [14]. This also could potentially increase the number of encounters healthcare providers' have with dogs in a clinical setting [16-18]. However, 16 states have made service dog fraud a criminal violation due to the rising number of incidents when poorly trained animals, incorrectly identified as service animals, have bitten people [10], attacked legitimate service dogs [11-13], and have been out of control [10]. Some service dog advocates worry that bad behavior by falsely labeled service dogs will increase bias and create a backlash against people with disabilities and mental illness, such as veterans, who qualify for a service animal [11, 12, 19].

In terms of access regulations, service dogs are allowed anywhere the public can go. This includes hospitals and clinics, which are required to meet requirements from the ADA [6]. However, after a review of its members' service dog policies, the Society for Healthcare Epidemiology of America (SHEA) concluded that healthcare institutions lacked consistent policies or practice around managing service dogs [20]. SHEA strongly recommended that medical facilities implement written policies addressing animals in a healthcare setting and educate their staff about the topic. The group outlined a list of specific policy guidelines to help institutions minimize risks and reduce the likelihood of an adverse event [21]. To our knowledge, no studies have subsequently examined the knowledge level of healthcare professionals about ADA regulations for service animals, which was the aim of our study.

\section{Methods}

This study was approved by the Ohio University Institutional Review Board.

The participant pool for this exploratory study included health professionals aged 18 years or older working in a clinic or hospital setting who could read or write English. Individuals working at long-term care facilities or in a home healthcare setting were excluded. Participants were recruited using mailing lists from the American Assembly for Men in Nursing as well as Ohio-licensed certified nurse practitioners, physician assistants, and physicians from the Ohio State Medical Board. Additionally, the research team also recruited participants via social media and in their own professional networks, including preceptors affiliated with the Ohio University Heritage College of Osteopathic Medicine, where the study took place. To encourage broader participation, respondents were asked to share the survey with colleagues (a "snowball sampling" method).

The anonymous, online survey was developed by the authors, with several survey questions based on previously published work on this topic [4]. The survey was administered through Qualtrics (SAP SE) between September and November 2018. Prospective participants followed a link to the survey, where they were presented with the informed consent form and asked to click a button affirming their consent. Once consent was given, participants could access the 24-item survey. The survey was divided into the following sections: questions regarding institutional policies, which assessed participants' comfort level with service dogs and awareness about policies and training related to service dogs; questions about ADA compliance, to examine their knowledge of federal regulations related to service dogs; and demographic questions about dog ownership, occupation, work facility, sex, and area of residence. Most questions used a 5-point Likert scale for answer options. There were multiple anchors, such as "extremely familiar" to "not familiar at all" or "strongly agree" to "strongly disagree," depending on the question type.

Before completing the ADA compliance section of the survey, participants were provided with the following definitions:

(1) Service dog-a dog that has been individually trained to do work or perform tasks for an individual with a disability. The task(s) performed by the dog must be directly related to the person's disability.

(2) Emotional support dog-a dog that provides comfort by being with the person but has not been trained to perform a specific job or task to assist people with disabilities.

(3) Therapy dog-a dog that provides comfort and affection to people, who may or may not have a disability, in schools, mental health institutions, health facilities, disaster areas and similar locations.

The questions in the compliance section then posed true/false questions to test knowledge; answers were coded as correct or incorrect. 
Most closed-end questions were analyzed using frequencies and descriptive statistics. As stated, true and false questions were used to test knowledge and then coded as correct or incorrect. A one-way analysis of variance (ANOVA) was conducted to test whether certain variables affected participants' knowledge of ADA service dog requirements. The relationship between having a pet and comfort level with service dogs was analyzed using Pearson chi-square with statistical significance defined as $\mathrm{p}<0.05$. Reliability and validity were not evaluated on the survey.

\section{Results}

The survey was completed by 441 health professionals, but not all participants responded to all questions; response rates and demographic information are shown in Table 1. Most participants were women (343; 77.8\%), lived in the Midwest (378; 90.6\%), and worked in a hospital (234; $53.1 \%)$ in either an urban $(211 ; 47.8 \%)$ or suburban (159; $36.1 \%)$ setting. Participants were primarily nurses (155; $35.2 \%)$, physicians $(71 ; 16.1 \%)$, or physician assistants $(65$; $14.7 \%)$, and $149(33.9 \%)$ participants worked in other health professions.

Although 318 (73.1\%) participants said their employer had a policy on service animals, 117 (26.9\%) said there was

Table 1: Participant demographics. ${ }^{a}$

\begin{tabular}{|c|c|}
\hline & n (\%) \\
\hline \multicolumn{2}{|l|}{ Sex, $n=438$} \\
\hline Women & $343(77.8)$ \\
\hline Men & $88(20.1)$ \\
\hline Prefer not to disclose & $7(1.6)$ \\
\hline \multicolumn{2}{|l|}{ Work setting, $n=438$} \\
\hline Urban & $211(47.8)$ \\
\hline Suburban & $159(36.1)$ \\
\hline Rural & $68(15.4)$ \\
\hline \multicolumn{2}{|l|}{ Region, $n=417$} \\
\hline West & $21(5.0)$ \\
\hline Midwest & $378(90.6)$ \\
\hline Northeast & $5(1.2)$ \\
\hline South & $13(3.1)$ \\
\hline \multicolumn{2}{|l|}{ Profession, $n=440$} \\
\hline Nurse & $155(35.2)$ \\
\hline Physician & $71(16.1)$ \\
\hline Physician assistant & $65(14.7)$ \\
\hline Other & 149 (33.9) \\
\hline \multicolumn{2}{|l|}{ Facility, $n=440$} \\
\hline Hospital & $234(53.1)$ \\
\hline Health clinic & $141(32.0)$ \\
\hline Rehabilitation center & $8(1.8)$ \\
\hline Other & $57(12.9)$ \\
\hline
\end{tabular}

${ }^{a}$ While the survey included 441 participants, many did not respond to all questions. Individual $\mathrm{n}$ values are indicated on the leading row for each category. no policy. Of those whose workplace had a policy, only 68 (20.9\%) said they were extremely or very familiar with that policy, 143 (44.1\%) had some familiarity, and 113 (34.9\%) were not familiar at all with the policy (Table 2). When asked if their employer had adequate protocols for managing service animals, 85 (19.6\%) strongly agreed and 106 $(24.5 \%)$ somewhat agreed. More than half of participants strongly agreed $(129 ; 29.7 \%)$ or somewhat agreed (122; $28.1 \%$ ) that their workplace provided adequate support for individuals with service animals. However, 236 (54.5\%) did not feel they had received adequate training from their employer on how to handle patients or visitors with service animals; 68 (15.5\%) reported they probably would not and $27(6.2 \%)$ said they definitely would not know what to do if a service animal entered the workplace. Fifty-seven (13.0\%) participants said they had never encountered a

Table 2: Workplace policy, training, and support. ${ }^{a}$

n (\%)

Does your clinic/hospital/facility have a policy

on service animals? $n=435$

Yes

$318(73.1)$

No

$117(26.9)$

How familiar are you with that policy? $n=324$

Extremely familiar

$28(8.6)$

Very familiar

$40(12.3)$

Moderately familiar

$69(21.3)$

Slightly familiar

$74(22.8)$

Not familiar at all

$113(34.9)$

My clinic/hospital has adequate protocols to address

how to manage service animals, $n=433$

Strongly agree

$85(19.6)$

Somewhat agree

$106(24.5)$

Neither agree nor disagree $156(36)$

Somewhat disagree $\quad 48(11.1)$

Strongly disagree

$38(8.8)$

My clinic/hospital provides adequate support

for individuals with service animals, $n=434$

Strongly agree

$129(29.7)$

Somewhat agree

$122(27.7)$

Neither agree nor disagree

Somewhat disagree

$136(30.8)$

$30(6.8)$

Strongly disagree

17 (3.9)

My clinic/hospital offers adequate training for

employees about handling patients/visitors

with service animals, $n=433$

Strongly agree $\quad 34(7.9)$

Somewhat agree $\quad 55(12.7)$

Neither agree nor disagree $108(24.9)$

Somewhat disagree $138(31.9)$

Strongly disagree $\quad 98(22.6)$

${ }^{a}$ While the survey included 441 participants, many did not respond to all questions. Individual $\mathrm{n}$ values are indicated on the leading row for each category. 
Table 3: Participant results for true/false knowledge-based questions regarding compliance $(n=441)$.

\begin{tabular}{|c|c|c|c|}
\hline Questions & Correct, n (\%) & Incorrect, n (\%) & Not sure, $\mathrm{n}(\%)$ \\
\hline $\begin{array}{l}\text { 1. Clinics/hospitals are required to allow } \\
\text { access to service dogs accompanying a patient or visitor. }\end{array}$ & $402(91.2 \%)$ & $5(1.1 \%)$ & $34(7.7 \%)$ \\
\hline $\begin{array}{l}\text { 2. Clinics/hospitals cannot restrict service dogs from entering } \\
\text { any area of the facility. }\end{array}$ & $180(40.8 \%)$ & $180(40.8 \%)$ & $81(18.4 \%)$ \\
\hline $\begin{array}{l}\text { 3. I can ask individuals with service dogs to provide proof } \\
\text { of the disability. }\end{array}$ & $199(45.1 \%)$ & $102(23.1 \%)$ & $140(31.7 \%)$ \\
\hline 4. I can ask for proof of a dog's status as a service dog. & $72(16.3 \%)$ & $268(60.8 \%)$ & $101(22.9 \%)$ \\
\hline $\begin{array}{l}5 . \text { I can ask individuals with service dogs if the dog is required } \\
\text { because of a disability. }{ }^{\text {a }}\end{array}$ & $160(36.3 \%)$ & $165(37.4 \%)$ & $115(26.1 \%)$ \\
\hline $\begin{array}{l}\text { 6. If a service animal is disruptive or uncontrollable, I can ask } \\
\text { that the dog be removed. }{ }^{\text {a }}\end{array}$ & $285(64.6 \%)$ & $30(6.8 \%)$ & $125(28.3 \%)$ \\
\hline 7. A pit bull cannot be a service dog. ${ }^{a}$ & $187(42.4 \%)$ & $43(9.8 \%)$ & $210(47.6 \%)$ \\
\hline
\end{tabular}

${ }^{a}$ Indicates missing data from participant nonresponse $(n=440)$.

service animal at work, while a similar number (53; $12.1 \%)$ reported frequently seeing service animals. Three hundred and seventy participants (83.9\%) said they were comfortable with service animals in the workplace. Two hundred and ninety-seven (67.3\%) participants had a dog as a pet, but very few had a service $(2 ; 0.5 \%)$, emotional support $(7 ; 1.6 \%)$, or therapy $\operatorname{dog}(7 ; 1.6 \%)$. No statistically significant relationship was found between participants having a pet or service dog and their comfort level when dealing with service dogs $(\mathrm{p}=0.110)$.

Only two of the true/false questions were correctly answered by most participants. Full results from these seven dichotomous variable questions are shown in Table 3. Almost all participants (402; 91.2\%) knew that clinics and hospitals are required to allow access to service dogs accompanying a patient or visitor. However, more participants either answered incorrectly or were unsure about specific questions about what is allowed. Two hundred and ten (47.6\%) of participants were unsure about whether a pit bull could be a service dog, 180 (40.8\%) incorrectly said service dogs cannot be restricted from entering any part of the facility, and 268 (60.8\%) incorrectly said they could ask for proof of a dog's status as a service dog.

A one-way ANOVA test was conducted to assess whether knowledge of ADA service dog requirements was affected by the frequency of encounters with service $\operatorname{dogs}(F[4,434]=3.486 ; \mathrm{p}=0.008)$, familiarity with service $\operatorname{dog}$ policies $(F[4,319]=4.613 ; \mathrm{p}=0.001)$, reports of adequate training $(F[4,428]=5.160 ; \mathrm{p}=0.000)$, confidence $(F[4,433]=7.568 ; \mathrm{p}=0.000)$, and comfort level $(F[4,436]=$ 2.763; $\mathrm{p}=0.027$ ) when dealing with service animals. The test revealed that knowledge scores were statistically significantly lower for the following: participants who had never encountered a service dog (mean \pm standard deviation [SD], $43.1 \pm 24.4 ; \mathrm{p}=0.011$ ), those who strongly disagreed when asked if they'd had adequate training (mean \pm SD, $43.3 \pm 24.7 ; \mathrm{p}=0.008$ ), and those who said they would not know what to do if a service dog entered their workplace (mean $\pm S D, 32.8 \pm 27.4 ; p=0.000)$. On the other hand, those who said they were extremely familiar with service dog policies had statistically significant higher (mean $\pm \mathrm{SD}, 60.7 \pm 21.9 ; \mathrm{p}=0.002)$ knowledge scores. Comfort level had no statistically significant effect on knowledge scores except for those who reported being neither comfortable nor uncomfortable with service animals in a professional setting. Their knowledge score was significantly lower (mean $\pm S D, 35.7 \pm 25.1 ; p=0.019)$. There was also a statistically significant difference between groups $(F[4,427]=5.906, \mathrm{p}=0.000)$ on knowledge scores among those who strongly agreed that they knew the difference between service animals, therapy animals, and emotional support animals, and those who were neutral $(\mathrm{p}=0.011)$, somewhat disagreed $(\mathrm{p}=0.002)$, or strongly disagreed $(\mathrm{p}=0.025)$.

\section{Discussion}

In this study, we sought to understand health professionals' knowledge about ADA service animal regulations. Medical facilities are likely to encounter service dogs and it is recommended that they have a policy regarding them [21]. We found that while many participants said their workplaces had a policy for service dogs, over a third (34.9\%) of participants said they were unfamiliar with the policy. Over half $(54.5 \%)$ of participants did not feel that they had received adequate training from their employers on handling patients or visitors with service animals. Our exploratory study results also showed that there was a lack of knowledge about service animals and ADA regulations, 
with the exception of participants who had reported that they were extremely familiar with their workplace's policy on service dogs. Of the seven questions given to participants on ADA requirements for service dogs, $5(71.4 \%)$ were answered incorrectly by most participants. Outside of knowing that service dogs can accompany patients, participants had a strong misunderstanding of regulations. For example, more than $57 \%$ of participants either were uncertain or mistakenly answered that pit bulls cannot be service dogs. The ADA states that any breed of dog may be a service animal [22].

Further, $40.8 \%$ of participants incorrectly answered that service animals cannot be restricted from some parts of a hospital. Although service animals can accompany a patient into an exam room and places where the public and patients are permitted, they can be excluded from areas where a sterile environment is required, food and medication are prepared, or where the animal poses a risk to immunocompromised patients [20]. If the service animal stays with patients when they are admitted to the hospital, the patient or their family and friends must be able to care for the dog. Otherwise, the hospital may place the dog in a boarding facility [22].

Nearly one-third (31.7\%) of participants were unsure whether they could ask patients to provide proof of their disability, and $60.8 \%$ incorrectly answered that they could ask for proof of the dog's status as a service animal. The ADA explicitly states that staff can ask two questions of service dog owners: (1) whether the dog a service animal required because of a disability, and (2) what work or task has the dog been trained to perform [6]. If the patient answers these questions, the animal must be given access. Owners cannot be asked about their disability or requested to show medical documentation or dog training certification. Staff cannot ask the person to state or describe their disability or ask for the dog to demonstrate the task it performs. They also cannot ask the animal to leave because staff can provide the same services or because staff or other patients are afraid or allergic to dogs. However, service dogs must meet local animal control or public health requirements, such as being vaccinated or licensed [22].

Many participants either incorrectly answered (30; $6.8 \%)$ or were unsure $(125 ; 28.3 \%)$ about whether service animals could be removed for being disruptive or uncontrolled. The ADA clearly states that staff may ask for service animals to be removed if the animal is out of control, not housebroken, disruptive, ill, or a direct threat to others' health and safety [22]. In most cases, the dog must be on a leash or tether and should not bark repeatedly [6].

Typically, service dogs are highly trained to help those with disabilities perform a specific task much like a medical device, such as an insulin pump or walker, but the ADA does not require the dog to be professionally trained nor wear a vest to indicate their status [22]. ADA knowledge was lower among participants who had not encountered service dogs, felt they had inadequate training, or said they were uncomfortable dealing with service dogs. Only participants who said they were extremely familiar with their workplace's policy and those who strongly agreed that they knew the difference between service animals and other classes of animals scored higher on the knowledge test than those who disagreed. These results suggest that increased of familiarity and training leads to higher knowledge levels about service dogs and ADA regulations.

Noncompliance with federal, state, and local regulations - such as refusing patients with service animals access to areas where members of the public are allowed to go - could result in patients taking legal action. Patients can file Title III complaints, which cover places of public accommodations, through private lawsuits in federal court or with the U.S. Department of Justice [23]. If the violation involves a federal government facility, activity, or program, or an organization receiving federal funds, the patient can file a section 504 complaint, which is done through the agency overseeing the funding or program [23].

A list of ADA cases since 2006 related to public access of service animals mostly contains complaints filed against schools, restaurants, hotels, and transportation companies [24]. However, healthcare facilities have also been the subject of complaints, lawsuits, and negative publicity for denying service animals access to facilities [25-27]. In one case [28], medical staff at a clinic complained about a patient's service animal and inappropriately asked for written proof of the dog's training and certification. The embarrassed patient left without being seen. The United States Attorney's Office conducted an investigation and found the office had violated the ADA. Consequently, the physician had to create a written service animal policy, distribute the policy to all employees, train them on it, integrate compliance with the policy into employee contracts, post signage saying individuals with service dogs were welcome in the office, promptly investigate complaints, respond in writing to individuals making complaints, record violations of the policy, and submit a biannual report of complaints for three years. He also had to pay $\$ 500$ in compensatory damages [28].

One recommendation to help healthcare organizations better manage service dogs in their facilities is to hire a liaison who would handle incident reports involving animals and act as a checkpoint for animals entering the building [21]. The results of our study suggest that it may be beneficial for hospital administrators to review their workplace's policies regarding service dogs and evaluate 
their employees' familiarity with them. The Society of Healthcare Epidemiology has published a thorough list of recommended service dog guidelines [21] addressing a broad range of topics such as infection control, interactions with animals, and policy development, which could be helpful for creating or updating policies. We also recommend that practices and health systems integrate service dog guidelines into their compliance manual and require employees to routinely review the policy, including the two questions that can be asked of service dog owners.

There are two primary limitations of our study, which include survey construction and generalizability of the results. This is among of the first studies investigating this topic as its primary purpose. Therefore, we based several of the survey questions on the limited work that was done previously [4]. Reliability and validity were not evaluated on the survey. It is unclear whether these trends are influenced by the environment, participants' perceptions, or participant background. In addition, use of a nonrandom sample is a potential limitation of the study. Although it can be useful for exploratory studies, it can also lead to oversampling, selfselection bias, and lack of a representative sample of the population.

We also did not consider specific state laws applicable to service dogs, nor how regional culture and attitude toward animals in public might influence health professionals' knowledge of service animals. Most study participants lived in the Midwest; a broader distribution of participants from around the country may have shown variations in knowledge by region.

\section{Conclusions}

Healthcare professionals who participated in our survey reported considerable uncertainty about and had received what they considered inadequate training on ADA service dog regulations, suggesting they could benefit from training to reduce safety risks and optimize health outcomes for individuals with service dogs. Developing a greater understanding of service dogs, their function in the overall health of patients who need them, and policies concerning service dog use in clinical settings is consistent with osteopathic philosophy and belief that good health extends beyond treating symptoms. Additionally, sensitivity to their use may help foster holistic and compassionate care based on the tenets of osteopathy. Future research examining the perceptions of patients with service dogs regarding their treatment and care at healthcare facilities could help identify gaps in how service dog policies are implemented.
Acknowledgments: The authors thank Brian Plow, MFA, and Tracy Shaub, DO, for their contributions to the study. Mr. Plow provided direct insight into service animal etiquette as a result of his experience as a documentarian currently working on a film about veterans and their relationship with service animals. Dr. Shaub provided support as Chair of Primary Care and with recruitment and conceptualization.

Research funding: None reported.

Author contributions: All authors provided substantial contributions to conception and design, acquisition of data, or analysis and interpretation of data; all authors drafted the article or revised it critically for important intellectual content; all authors gave final approval of the version of the article to be published; and all authors agree to be accountable for all aspects of the work in ensuring that questions related to the accuracy or integrity of any part of the work are appropriately investigated and resolved.

Competing interests: Authors state no conflict of interest. Informed consent: Informed consent was obtained from all survey participants in this study.

Ethical approval: This study was approved by the Ohio University Institutional Review Board.

\section{References}

1. Winkle M, Crowe TK, Hendrix I. Service dogs and people with physical disabilities partnerships: a systematic review. Occup Ther Int 2012;19:54-66.

2. Hall SS, MacMichael J, Turner A, Mills DS. A survey of the impact of owning a service dog on quality of life for individuals with physical and hearing disability: a pilot study. Health Qual Life Outcome 2017;15:1-9.

3. Crowe TK, Perea-Burns S, Sedillo JS, Hendrix IC, Winkle M, Deitz J. Effects of partnerships between people with mobility challenges and service dogs. Am J Occup Ther. 2014;68:194-202.

4. Schoenfeld-Tacher R, Hellyer P, Cheung L, Kogan L. Public perceptions of service dogs, emotional support dogs, and therapy dogs. Int J Environ Res Publ Health 2017;14:642.

5. Walther S, Yamamoto M, Thigpen AP, Garcia A, Willits NH, Hart LA. Assistance dogs: historic patterns and roles of dogs placed by ADI or IGDF accredited facilities and by non-accredited U.S. Facilities. Front Vet Sci 2017;4:1.

6. U.S. Department of Justice. ADA requirements: service animals. Available from: https://www.ada.gov/service_animals_2010.htm [Accessed 4 Oct 2017].

7. Freedom Service Dogs of America. FAQs: applying for a service dog. Freedom service dogs. Available from: https:// freedomservicedogs.org/our-dogs/faqs-applying-for-a-servicedog/ [Accessed 9 Oct 2017].

8. American Veterinary Medical Pet Association. AVMA Ownership and Demographics Sourcebook: 2017-2018 Edition; 2018. Available from: https://ebusiness.avma.org/ProductCatalog/ ProductCategory.aspx?ID=120 [Accessed 11 Dec 2020]. 
9. BarkBox. New study finds dogs make people better, happier and healthier humans. Kelton global. Available from: http:// keltonglobal.com/in-the-media/new-study-finds-dogs-makepeople-better-happier-healthier-humans/ [Accessed 9 Oct 2017].

10. Jonsson $P$. Is that Chihuahua a real service dog? The truth, unleashed. The Christian Science Monitor. Available from: https://www.csmonitor.com/USA/Society/2019/0808/ls-thatChihuahuaa-real-service-dog-The-truth-unleashed [Accessed 11 Dec 2020].

11. Weill K. Finally, Colorado is cracking down on service dog fraud. The Daily Beast. Available from: https://www.thedailybeast. com/finally-colorado-is-cracking-down-on-service-dog-fraud [Accessed 9 Oct 2017].

12. Maiman B. Frustrations of service dog fraud. The Sacramento Bee. Available from: http://www.sacbee.com/opinion/op-ed/ article2605800.html [Accessed 9 Oct 2017].

13. Manning S. Fake service dogs a growing problem - NBC News. Available from: https://www.nbcnews.com/health/fake-servicedogs-growing-problem-8c11366537 [Accessed 9 Oct 2017].

14. Herzog H. Service animal scams: a growing problem / psychology today. Available from: https://www.psychologytoday.com/blog/ animals-and-us/201406/service-animal-scams-growingproblem [Accessed 9 Oct 2017].

15. Appiah KA. Should I call my friend out for her 'service dog' scam? The New York Times. Available from: https://www.nytimes.com/ 2016/11/30/magazine/should-i-call-my-friend-out-for-herservice-dog-scam.html [Accessed 9 Oct 2017].

16. Pellegrino LD, Cerimele JM, Dubovsky AN. Service dogs in the hospital: helpful or harmful? A case report and clinical recommendations. Psychosomatics 2016;57:301-4.

17. McNary AL. "Vetting" service dogs and emotional support animals. Innovat Clin Neurosci 2018;15:49-51.

18. Levey JA, Chappy SL. Service dogs in the perioperative setting. AORN J 2017;105:365-9.

19. Lee T. Criminalizing fake service dogs: helping or hurting legitimate handlers. Anim Law 2016;23:325-54.
20. Lefebvre SL, Golab GC, Christensen E, Castrodale L, Aureden K, Bialachowski A, et al. Guidelines for animal-assisted interventions in health care facilities. Am J Infect Contr 2008;36: 78-85.

21. Murthy R, Bearman G, Brown S, Bryant K, Chinn R, Hewlett A, et al. Animals in healthcare facilities: recommendations to minimize potential risks | infection control \& hospital epidemiology | Cambridge core. Available from: https://www.cambridge.org/ core/journals/infection-control-and-hospital-epidemiology/article/ animals-in-healthcare-facilities-recommendations-to-minimizepotential-risks/7086725BAB2AAA4C1949DA5B90F06F3B [Accessed 3 Oct 2017].

22. U.S. Department of Justice. Frequently asked questions about service animals and the ADA. Available from: https://www.ada. gov/regs2010/service_animal_qa.html [Accessed 30 Aug 2019].

23. Brennan J. Service animals and emotional support animals |ADA National Network. Available from: https://adata.org/guide/serviceanimals-and-emotional-support-animals [Accessed 28 Jan 2020].

24. U.S. Department of Justice. ADA enforcement. Available from: https://www.ada.gov/enforce_current.htm\#Titlelll [Accessed 29 Jan 2020].

25. TAMARA v. EL CAMINO HOSP. | 964 F.Supp.2d 1077 (2013) | 20130805635 | Leagle.com. Leagle. Available from: https://www. leagle.com/decision/infdco20130805635 [Accessed 29 Jan 2020].

26. Roe v. PROVIDENCE HEALTH SYSTEM-OREGON, 655 F. Supp. $2 \mathrm{~d}$ 1164 - CourtListener.com. CourtListener. Available from: https:// www.courtlistener.com/opinion/2432827/roe-v-providencehealth-system-oregon/ [Accessed 28 Jan 2020].

27. Nieto-Munoz S. Hospital kicked out my service dog, threatened to send her to the pound, woman says. NJ.com. Available from: https://www.nj.com/news/2018/10/my_dog_detects_my_ seizures_but_hospital_threatened_to_send_him_to_the_ pound_woman_says.html [Accessed 28 Jan 2020].

28. Settlement agreement between the United States of America and Dr. Bruce Berenson M.D., P.A. Available from: https://www.ada. gov/berenson_settle.htm [Accessed 29 Jan 2020]. 